\title{
Urinary bladder cancer: biomarkers and target therapy, new era for more attention
}

\author{
Amrallah A. Mohammed, ${ }^{1,2}$ Hani EL-Tanni, ${ }^{2}$ Hani M. EL-Khatib, ${ }^{2}$ Ahmad A. Mirza, ${ }^{3}$ \\ Abdulrahim A. Mirza, ${ }^{4}$ Turki H. Alturaifi ${ }^{4}$ \\ ${ }^{1}$ Department of Medical Oncology, Faculty of Medicine, Zagazig University, Zagazig, Egypt; \\ ${ }^{2}$ Oncology Center, King Abdullah Medical City-Holy Capital, Makkah, Saudi Arabia; \\ ${ }^{3}$ Department of Surgery, College of Medicine, Taif University, Taif, Saudi Arabia; ${ }^{4}$ Faculty of \\ Medicine, Umm Al-Qura University, Makkah, Saudi Arabia
}

\begin{abstract}
Currently, bladder cancer (BCa) evaluation depends mainly on traditional clinicopathological parameters encompassing tumor stage and grade, which will not reflect the behavior of the disease. Diverse molecular alterations are responsible for the heterogeneous course. The differences in molecular pathogenesis between non-invasive $\mathrm{BCa}$ and invasive $\mathrm{BCa}$ have been recognized. Molecular biomarkers are promising to predict progression and survival. The management of advanced $\mathrm{BCa}$ remains somewhat primitive in comparison with other more common malignancies. This topic will discuss the molecular pathways, biomarkers and potential targets that may improve the outcome in BCa.
\end{abstract}

\section{Introduction}

Bladder cancer (BCa) is one of the most common cancers; an estimated 74,000 new cases diagnosed/year. It is three times more prevalent in men and is rarely diagnosed before 40 years old. The molecular heterogeneity of $\mathrm{BCa}$ is believed to be one of the factors responsible for large variation in the response to treatment. ${ }^{1}$ The molecular diagnosis is now a part of clinical management for many types of cancers, however for $\mathrm{BCa}$ is still missing and has not achieved widespread clin-

Correspondence: Amrallah A. Mohammed, Oncology Center, King Abdullah Medical City-Holy Capital, Muzdallifa Street, P.0. Box 57657, 21995 Makkah, Saudi Arabia.

Tel.: +96.6566979027 .

E-mail: amrallaabdelmoneem@yahoo.com

Key words: Bladder cancer; targeted therapies; biomarkers.

Received for publication: 13 October 2016 .

Revision received: 23 November 2016.

Accepted for publication: 24 November 2016.

This work is licensed under a Creative Commons Attribution NonCommercial 4.0 License (CC BY-NC 4.0).

(C) Copyright A.A. Mohammed et al., 2016

Licensee PAGEPress, Italy

Oncology Reviews 2016; 10:320

doi:10.4081/oncol.2016.320 ical use. ${ }^{2}$ Over the last 10 years, significant progress had been made in the era of molecular diagnosis and target therapy aiming to improve the quality of life. The high frequency of recurrence of non-invasive $\mathrm{BCa}$ and poor survival rate of invasive $\mathrm{BCa}$ emphasize the need for novel therapeutic approaches. In this review, we describe the molecular basis, biomarkers, and the possible targeted agents that may improve the outcome of $\mathrm{BCa}$.

\section{Molecular basis of bladder cancer}

The transition from normal urothelial tissue to carcinoma is associated with mainly two divergent major molecular events, called noninvasive papillary urothelial $\mathrm{BCa}$ (superficial) and non papillary invasive or solid $\mathrm{BCa} .^{3}$ Papillary or superficial tumors are believed to be originated from diffuse flat hyperplastic urothelial changes, typically, characterized by low histological grade without basement membrane (BM) or muscle layer invasion. Clinically, they have a high recurrence rate with low probability (10-15\%) to progress to histological high grade. ${ }^{4}$ FGFR-3, H-RAS, and PI3KCA are the main genetic alternations involved in its development. Non-papillary invasive tumors develop from severe dysplasia or carcinoma in situ (CIS), characterized by high histological grade with high penetration affinity either to BM (T1) or to muscle layer (T2). Clinically, they have an aggressive behavior with tendency for metastasis either to regional lymph nodes or distance spread. ${ }^{5}$ Molecular alterations are mainly characterized by mutations in tumor suppressor genes (TSGs) mainly, p53, and Rb. ${ }^{6}$ Figure 1 illustrates the molecular pathways of $\mathrm{BCa}$; papillary (superficial) and invasive types.

\section{Biomarkers}

The National Cancer Institute defines a biomarker as a substance found in tissue, blood, or other body fluids that may be a sign of cancer or noncancerous conditions. Most tumor biomarkers are secreted by both normal cells as well as cancer cells, but in different amounts. Like all respective biomarkers related to their cancers, BCa biomarkers exhibit some or all of these features: prognostic, predictive, and pharmacodynamic. Prognostic biomarkers predict the natural course of the cancer. Predictive biomarkers evaluate the probable benefit of a particular treatment. Pharmacodynamic biomarkers assess the treatment effects of a drug on a tumor and can possibly determine the proper dosage of a new anticancer drug. ${ }^{7}$ Identification of disease-specific 
molecular pathogenesis and biomarkers are a rational approach to better assist in the clinical management. In superficial tumors, identify who will be in need for close observation and vigilant surveillance. Furthermore, the poor prognosis of invasive $\mathrm{BCa}$; the biomarkers may improve the prognostication (Figure 2).

\section{Chromosomal changes}

Cytogenetic studies identified several patterns of chromosomal changes, which have been implicated in BCa management. These aberrations can be detected by multicolor interphase fluorescence in situ hybridization (FISH), single nucleotide polymorphism analysis (SNP), or comparative genomic hybridization (CGH). Chromosome 9 alterations (9q-, 9p-) are the earliest genetic events in both superficial and muscle-invasive $\mathrm{BCa} .{ }^{8}$ This is corresponding to several previous studies. $^{9-11}$

\section{Cell cycle regulators}

Altered cell cycle regulations have been extensively examined in BCa. Missense mutations in TP53 with subsequently nuclear accumu- lation of p53 leading to cell cycle deregulation progression through the G1-S checkpoint. ${ }^{12}$

Early studies on the prognostic value of p53 expression revealed the association with higher rate of distant metastasis and reduced overall survival. ${ }^{13}$ Similarly, many studies have postulated that an altered p53 status may predict a poor outcome in BCa patients, after surgical treatment. ${ }^{14}$ However, substantial controversy exists regarding this prognostic value. ${ }^{15}$ Actually, there are inconclusive and variable results regarding the prognostic value of p53. This controversy may arise out of the heterogeneity of the targeted patients, methodologies or evaluation criteria. P21 and p27 are G1-checkpoint CDK inhibitors, has been implicated in the genesis or progression of many human malignancies. A meta-analysis was performed in 48 patients with $\mathrm{BCa}$ revealed that low p21 expression predicted tumor recurrence and poor prognosis, ${ }^{16}$ which is corresponding to the study done by Korkolopoulou et al. ${ }^{17}$

A systematic review and meta-analysis done by Gan et al., suggested that p16, encoded by the $C D K N 2 A$ gene, might play an essential role in the deterioration of $\mathrm{BCa}$ and could serve as a biomarker for the prediction for patients' progression and prognosis. ${ }^{18}$ Although the data regarding the prognostic value of cyclin D1 is equivocal, a subgroup analysis in a study of $150 \mathrm{BCa}$ patients revealed that over expression is considered as a predictor for lower progression rate and eventually better survival. ${ }^{19}$ Cox's multivariate analysis selected cyclin D3 as an independent predictor of progression-free survival together with tumor size in patients with $\mathrm{Ta} / \mathrm{T} 1 \mathrm{BCa} .^{20} \mathrm{~A}$ study of protein levels of cyclin $\mathrm{E}$ and p27 levels in BCa demonstrated significantly improved survival associ-

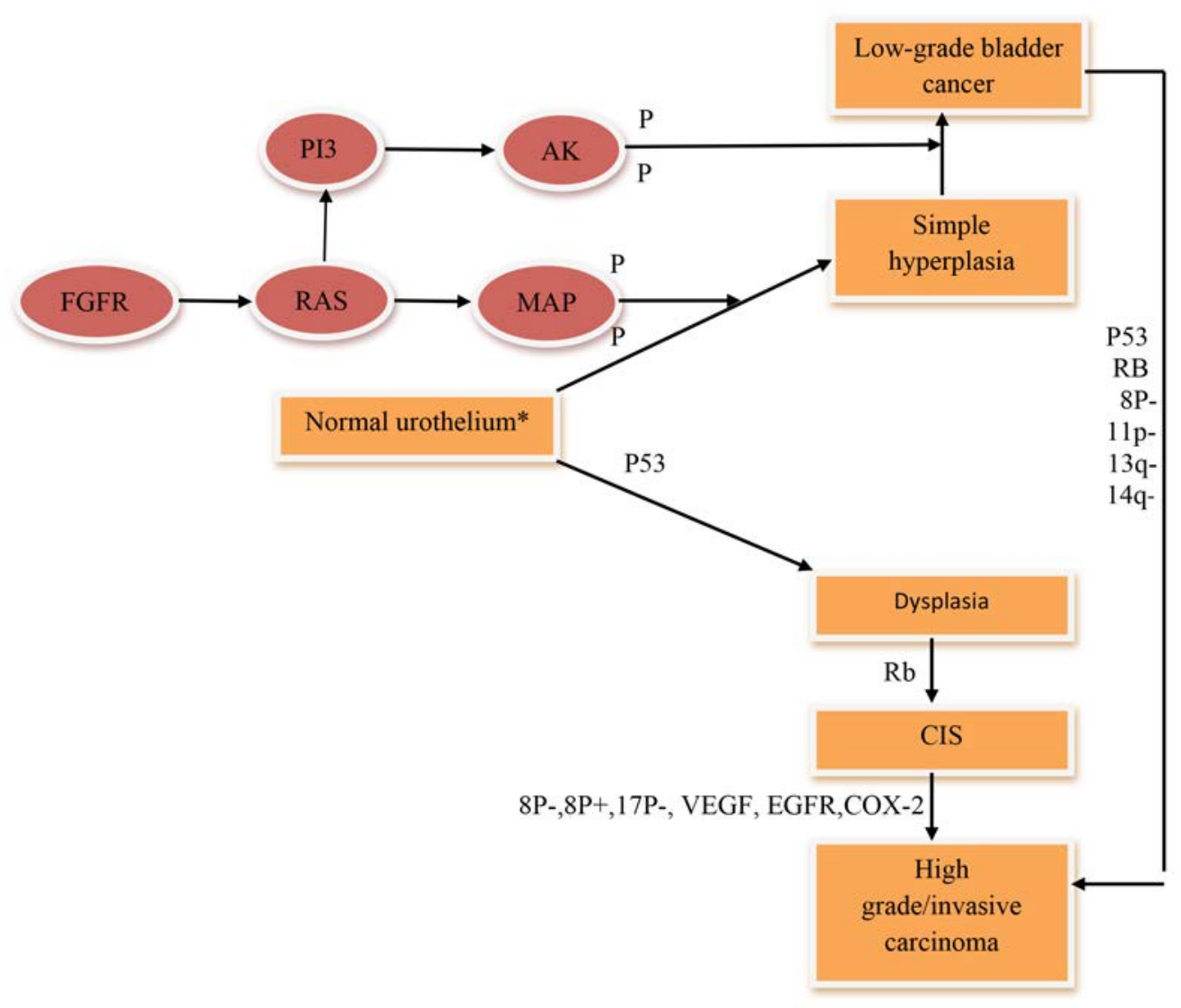

*Chromosome 9 alterations (9q- , 9p-) are the earliest genetic events in both superficial and muscle-invasive $\mathrm{BCa}$.

Figure 1. The molecular pathways of bladder cancer; papillary (superficial) and invasive types. 
ated with low protein expression. ${ }^{21}$ The retinoblastoma gene $(\mathrm{Rb})$ is a TSG that regulates cell-cycle progression at the G1-S transition. The RB gene inactivation was associated with high grade $\mathrm{BCa}$, surprisingly; both high and low Rb levels correlate with outcome. This may be explained by discordance between proteins over expression and gene mutation. $^{22}$

\section{Proliferation markers}

Carcinogenesis is a multistage process initiated by disturbed and uncontrolled proliferation of cells. Their growth is associated with the actions of such proliferative proteins as proliferating cell nuclear antigen (PCNA), Ki-67 and MCM-2 (minichromosome maintenance 2). In multivariate Cox regression analysis, the expression of $\mathrm{Mcm} 2$ with a cutoff $\geq 40 \%$ was independent predictor of recurrence rate in $\mathrm{Ta} / \mathrm{T} 1$ BC. ${ }^{23}$ The Ki-67 labeling index is a widely used immunohistochemical marker for determining the proliferative activity of various tumors. Elevated Ki-67 labeling index has both prediction and prognostic characteristics in BCa. In a study done by Margulis et al., elevated Ki-67 labeling index had associated with poor outcome in form of diseasespecific mortality and high probabilities of recurrence, ${ }^{24}$ and in another study by Ding et al., identified the improved the prediction of both progression free survival and disease specific survival when combined Ki-
67 with European Organization for Research and Treatment of Cancer (EORTC) risk scores. ${ }^{25}$ In addition, the PCNA over expression was associated with micro vessel density (MVD) may predict the survival. ${ }^{26}$

\section{Apoptosis markers}

Inappropriate apoptosis is a characteristic of many types of human cancer. Bcl-2, caspase-3, P53, and survivin have a cooperative effect on progression of $\mathrm{BCa}$. By univariate and multivariate analysis of $226 \mathrm{BCa}$ patients, altered expression of Bcl-2, caspase-3, P53, and survivin were independently all associated with high probability of disease recurrence and disease-specific mortality. ${ }^{27}$ The value of Bcl-2/Bax expression ratio in $\mathrm{BCa}$ was evaluated by Bahram et al., concluded that this expression may serve as a significant prognostic indicator in predicting the clinical outcome. ${ }^{28}$ Immunohistochemical analysis of Bcl-xL expression revealed that patients with a high Bcl-xL score had a significantly lower 5-year cancer-specific survival rate (53.2\%) than those with a low Bcl-xL score (77.2\%), and servants as an independent prognostic factor through multivariate analysis. ${ }^{29}$ An active caspase-3 is a common finding in $\mathrm{BCa}$ with protein over expression was associated with better survival indicating that, it might be a relevant prognostic factor for BCa patients. ${ }^{30}$

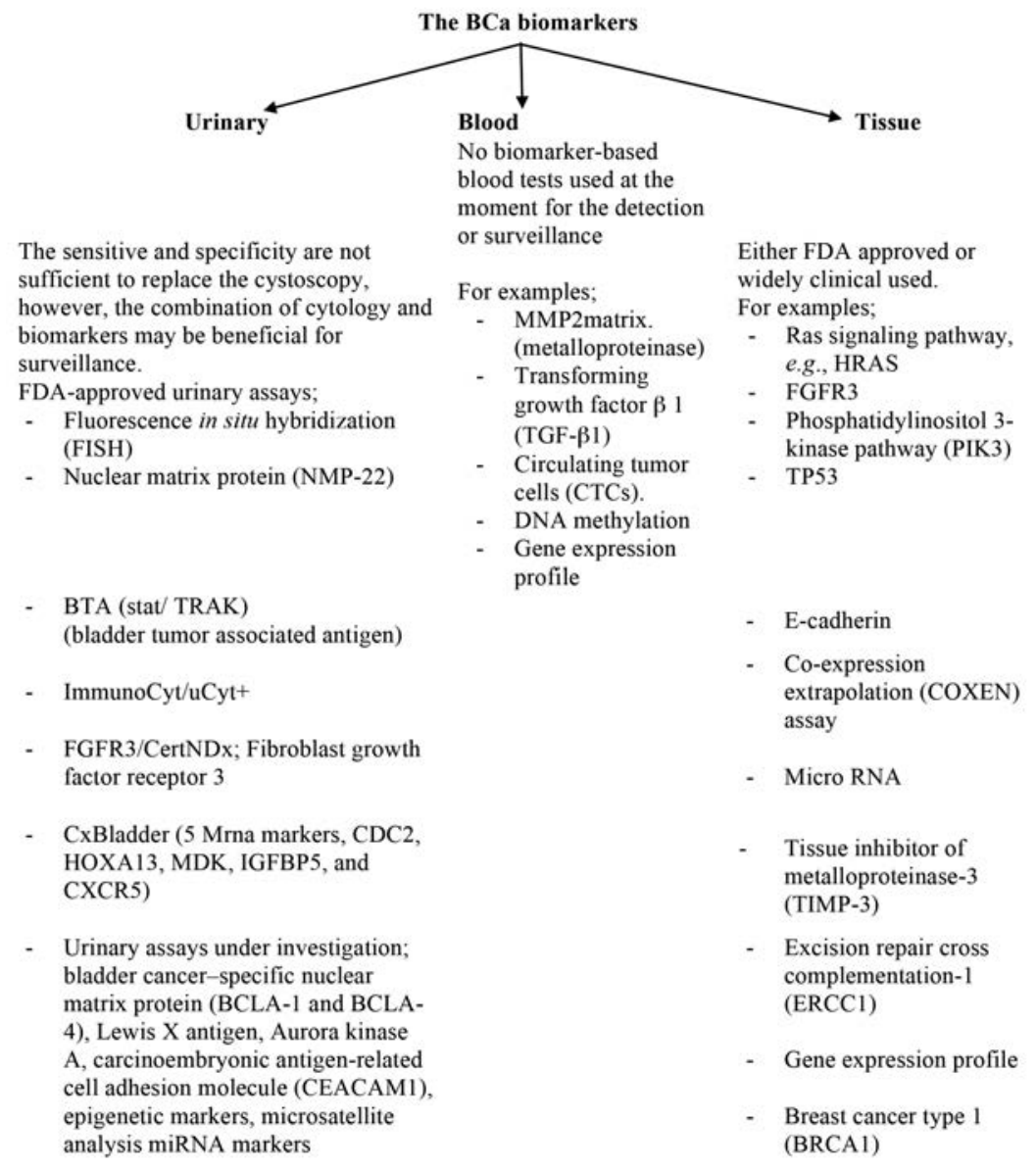

Figure 2. The subdivision of bladder cancer biomarkers. 


\section{Tyrosine kinase-associated receptors}

Several cell growth receptors and their downstream pathways have emerged as key regulators of many cellular processes. Any changes in these receptors or their signals can lead to uncontrolled cellular growth and tumorogenesis. There are four-fibroblast growth factor receptors (FGFR1-4) genes result in the production of over 48 different isoforms of FGFR. Among FGFR family, FGFR3 is the most extensively studied alterations. As mentioned before, FGFR3 mutations and protein over expression are associated with papillary low-grade tumors. FGFR3 mutations have been evaluated as their prognostic significance. ${ }^{31}$ FGFR3 over expression was predictive of adverse outcome in patients with adjuvant cisplatin-based chemotherapy after radical cystectomy. ${ }^{32}$ In the stratified analysis of 722 patients with non invasive $\mathrm{BCa}$, only patients with FGFR3 mutations TaG1 tumors have a higher risk of recurrence. ${ }^{33}$ In a multicenter study, included 286 patients with primary BCa, FGFR3 status and MIB-1 were evaluated with mean followup was 5.5 years identified this combination may be considered independently prognostic significant. ${ }^{34}$

The epidermal growth factor receptor (EGFR) belongs to the ErbB family of receptor tyrosine kinases. EGFR (ErbB-1) and ErbB-2 (Her2/neu) are among the best-studied receptors in this family. EGFR over expression is an independent predictor of poor survival and stage progression. It was found to be $80 \%$ sensitive in predicting stage progression in non- invasive high grade $\mathrm{BCa} .{ }^{35}$ Based on multivariate Cox regression analyses, Kim et al., demonstrated that two-gene risk score (composed of EGFR and S100A9) was the only factor that was independently prognostic for disease progression. ${ }^{36}$ The mutation assays for the detection of 19 possible mutations in the HRAS, KRAS, and NRAS genes on 257 patients with primary BCa and 184 recurrences revealed that mutations in the RAS and PIK3CA genes were not predictors for recurrence-free (RFS), progression-free (PFS) and diseasespecific survival (DSS). ${ }^{37}$

\section{Epigenetic markers}

Although only few data are available, the significant association between hypermethylation of the promoter region and decreased survival in BCa has been reported. Some studies have shown a positive correlation between hypermethylation status of genes (e.g., DAPK, p14ARF, TIMP-3 and RUNX3) and high recurrence rate of BCa, and some of these were independent predictive factors for recurrence. ${ }^{38}$ In a study, methylation status of ten genes was determined in $98 \mathrm{BCa}$ specimens and methylation index was calculated and demonstrated that an association between methylation of $\mathrm{CDH} 1$ and survival. ${ }^{39}$ In addition, the methylation status of 20 cancer-associated genes for DNA methylation in 105 consecutive patients with primary non invasive $\mathrm{BCa}$ were evaluated and concluded that, the methylation status of six genes (SOCS-1, STAT-1, BCL-2, DAPK, TIMP-3 and CDH1) was associated with tumor recurrence. ${ }^{40}$ Moreover, in another study, the roles of the methylation of 25 TSGs as clinical outcome predictive biomarkers in T1G3 Bca treated with BCG were evaluated. Multivariate analyses demonstrated the association between the methylation status of TSGs and the clinical outcome of T1G3 tumors treated with BCG through three clinical end points: RFS, PFS, and DSS. ${ }^{41}$

\section{Invasion and angiogenesis markers}

Recent studies have pointed out the potential prognostic value of angiogenesis and invasion markers in BCa. In Cox univariate analysis revealed that the decrease of $\mathrm{E}$-cadherin and the gain of $\mathrm{N}$-cadherin gene expression were considered as risk factors for cancer-related death. ${ }^{42}$ Results from another study revealed the association between decreased E-Cadherin immunoexpression and tumor recurrence in low-grade and non invasive $\mathrm{BCa} .{ }^{43}$ Several matrix metalloproteinases (MMPs) have been identified as having potential diagnostic or prognostic utility, whether alone or in combination with cytology. ${ }^{44}$

The prognostic significance of the hepatocyte growth factor (HGFR) was evaluated in 133 patients with non-metastatic $\mathrm{BCa}$ and postulated that the phosphorylated form was an independent predictor of RFS and DSS on multivariable analysis. ${ }^{45}$

The expressions of VEGF, VEGFR1, and VEGFR2 in non-invasive and invasive BCs were investigated and identified that, patients with higher levels of VEGF, VEGFR1, and VEGFR2 tended to have poorer RFS than those with lower levels, but this was not statistically significant. ${ }^{46}$ Moreover, the correlation MVD with grade and stage of BCa were indentified in another study. ${ }^{47}$

\section{Target therapy}

Targeted therapy is used to keep cancer from growing and spreading. They are often able to attack cancer cells while doing less damage to normal cells. Due to the high probability of recurrence of noninvasive $\mathrm{BCa}$ and poor survival rate of invasive $\mathrm{BCa}$, new therapeutic approaches are needed. A cancer growth and spread are related to many growth factors pathways including the fibroblast, epidermal, and the vascular endothelial growth factor pathways.

\section{Fibroblast growth factor receptor}

Although the role of fibroblast growth factor receptor (FGFR) has been documented in pathogenesis of low grade non-invasive $\mathrm{BCa}$, yet clinical benefit of its inhibition is not yet known. Gust et al., evaluated the role of FGFR3 inhibition with R3Mab in patients with $\mathrm{BCa} .{ }^{48}$ The pharmacodynamics, efficacy and safety of dovitinib-a broad multikinase inhibitor of FGFR, VEGFR and PDGFR including FGFR3-were evaluated in patients with previously treated advanced urothelial carcinoma (UC), with and without FGFR3 mutations. Although generally well tolerated, dovitinib has very limited single-agent activity regardless of FGFR3 mutation status. ${ }^{49}$

\section{Epidermal growth factor receptor}

\section{Gefitinib}

Gefitinib, an orally active selective epidermal growth factor receptor (EGFR) tyrosine kinase inhibitor (TKI), inhibits the receptor and its related downstream process. It was evaluated as a single agent in 31 patients with metastatic UC in platinum refractory chemotherapy. $81 \%$ of patients demonstrated progressive disease and the median survival time was 3 months. ${ }^{50}$

Moreover, the addition of gefitinib did not appear to improve the response rate or survival outcome in comparison with historical controls of patient treated just with cisplatin plus gemcitabine. ${ }^{51}$

\section{Erlotinib}

In a phase II open-label trial to evaluate Erlotinib as neoadjuvant; $60 \%$ were down staged suggesting single-agent activity with EGFR 
inhibitors in this disease setting. ${ }^{52}$ This is corresponding to the study at MD Anderson Cancer Center with complete pathologic response after 3 to 5 weeks of erlotinib. ${ }^{53}$

\section{Cetuximab}

Cetuximab is anti-EGFR monoclonal antibody has modest activity when used as monotherapy in advanced or metastatic $\mathrm{BCa}$, moreover, in a randomized phase II trial, the use of cetuximab with or without paclitaxel in 39 patients with previously treated UC showed that cetuximab has limited activity as a single agent. ${ }^{54}$

\section{Panitumumab}

Panitumumab, another anti-EGFR monoclonal antibody, was evaluated in combination with radiotherapy (RP/P) following neoadjuvant chemotherapy and pelvic lymph node dissection. The authors suggest that this combination is safe an organ preserving option for $\mathrm{BCa}^{55}$

\section{Human epidermal growth factor receptor 2}

\section{Trastuzumab}

Trastuzumab, the anti-Her-2 humanized monoclonal antibody was used in combination with gemcitabine with cisplatin or carboplatin to treat patients with advanced Her-2-positive BCa (arm A) or without (arm B) trastuzumab. Median PFS for arms A and B was 10.2 versus 8.2 months, ORR 65\% versus $53.2 \%$, and median OS 15.7 versus 14.1 months $(\mathrm{P}=0.684)$, respectively. However, the low number of patients with Her-2 positive limited the power of the study. ${ }^{56}$

\section{Lapatinib}

Lapatinib was evaluated as a second-line treatment of patients with locally advanced or metastatic UC in a single-arm, multicenter, openlabel phase II study revealed negative results because it did not meet its primary endpoint. ${ }^{57}$ The safety of lapatinib in combination with gemcitabine /cisplatin in patients with advanced BCa was demonstrated in phase II study done by Narayan et al., revealed the intolerability of this combination. ${ }^{58}$ Moreover, the use of maintenance lapatinib in Her1/2 positive metastatic BCa patients did not improve outcomes in HER1 or HER2 positive individuals versus placebo after first line chemotherapy. ${ }^{59}$

\section{Antiangiogenesis}

\section{Bevacizumab}

Bevacizumab, a monoclonal antibody against VEGF, has been utilized as neoadjuvant with chemotherapy in several nonrandomized phase II trials without down-staging of the tumor and higher rates of surgical complications. ${ }^{60}$ In the first-line setting, it was used in combination with gemcitabine and carboplatin in patients with advanced BCa but without satisfaction results. ${ }^{61}$ The other anti-angiogenic agents as, sunitinib, pazopanib, aflibercept and ramucirumab have been evaluated in multiple settings in UC, without clear clinical benefit. ${ }^{62-65}$

\section{Checkpoint inhibitors}

Atezolizumab belongs to a class of immunotherapy drugs known as checkpoint inhibitors. It prevents a programmed cell death (PD-L1) that is found on some tumor cells from binding to another protein, PD1 , on immune cells with subsequently immune response suppression. By this blocking the immune cells can attack the tumors. The Food and
Drug Administration (FDA) on May 18 2016, approved atezolizumab for the treatment of patients with UC, based on a study of 310 patients with locally advanced or metastatic UC after failure of platinum-containing chemotherapy or within one year of receiving it. In this trial, all patients received a 1200-mg intravenous dose of the atezolizumab on day 1 every 21-days until unacceptable toxicity or progression occurred. The objective response rate was $26 \%$ in patients with the highest positivity for PD-L1, 18\% for the lower positivity, and 15\% for all patients. Complete responses were seen in up to $11 \%$ of patients in the highestpositivity subgroup and in 5\% of all patients. Median PFS was 2.1 months for all patients. At 6 months, it was $30 \%$ in the highest-positivity subgroup, $17 \%$ in the lower-positivity subgroup, and $21 \%$ in the subgroup with no/minimal PD-L1 expression. Median OS was 7.9 months for all patients, 11.4 months for the highest-positivity subgroup, and 6.7 months for the lowest-positivity subgroup. The most common grade 3 4 adverse reactions ( $\geq 2 \%$ ) were urinary tract infection (9\%), anemia (8\%), fatigue (6\%), hematuria (3\%), and dyspnea (4\%) ${ }^{66}$ Several PD1 inhibitors as of pembrolizumab or nivolumab, are currently being studied in platinum-refractory metastatic BCa patients. ${ }^{67}$

\section{Others}

In a single-arm, nonrandomized, phase II study of everolimus in metastatic UC, yield one partial response, one near-complete response and twelve minor regressions were observed. ${ }^{68}$ Moreover, in a phase I trial, a BCa patient had a 14-month complete response with the combination of the mTOR inhibitor everolimus and pazopanib. It supposed that response was related to everolimus and not pazopanib treatment, because the dose of pazopanib needed to overcome the activity of the mTOR mutations is more than 100 -fold higher than that for rapamycin. ${ }^{69}$ Similarly, the use of single temsirolimus failed to show a considerable activity in advanced UC. ${ }^{70}$

Cabozantinib is TKI that primarily targets MET/HGF1. It was evaluated in phase II trial as second-line treatment of 26 patients with relapsed or refractory metastatic UC. The authors concluded that the cabozantinib had clinical activity in patients with relapsed or refractory metastatic UC with manageable toxicities. ${ }^{71}$

\section{Conclusions}

Nowadays many cancers cannot be managed according the pathological features only; we expect $\mathrm{BCa}$ is an important member from these cancers, which will include beside the clinicopathological features, the molecular-guided approach including several markers mentioned above. Although, urinary bladder biomarkers represent a promising frontier for predicting not only the course of the disease but also the response of that tumor to therapy, its use still immature and the routine use is not recommended.

Due to disease heterogeneity, there is no single marker has been able to adequately reflect the tumor biology. Moreover, the combining biomarkers had been found to improve the predictive and prognostic accuracy in many studies. We are in need to classify BCa into low risk and high risk based on molecular features to get the personalized treatment approach.

Although new combination chemotherapy protocols have improved median survival, there is still a great opportunity for improvement. Targeted agents are now emerging as a potential ways to improve the outcome. The response rates may be improved via refinement of the markers used to predict response.

After the approval of atezolizumab as single agent in advanced BCa, many of new targeted therapeutic agents are under investigation in combination with standard chemotherapy agents or combined checkpoint inhibitors either double or triple, which may move the manage- 
ment from the advanced platinum-refractory BCa to first line or even as a maintenance.

\section{References}

1. Siegel RL, Miller KD, Jemal A. Cancer statistics 2015. CA Cancer J Clin 2015;65:5-29.

2. Xylinas E, Kluth LA, Rieken M, et al. Urine markers for detection and surveillance of bladder cancer. Urol Oncol 2014;32:222-9.

3. Dinney CP, McConkey DJ, Millikan RE, et al. Focus on bladder cancer. Cancer Cell 2004;6:111-6.

4. Mc Conkey DJ, Lee S, Choi W, et al. Molecular genetics of bladder cancer: Emerging mechanisms of tumor initiation and progression. Urol Oncol 2010;28:429-40.

5. Millis SZ, Bryant D, Basu G, et al. Molecular profiling of infiltrating urothelial carcinoma of bladder and non bladder origin. Clin Genitourin Cancer 2015;13:e37-49.

6. Knowles MA, Hurst CD. Molecular biology of bladder cancer: new insights into pathogenesis and clinical diversity. Nat Rev Cancer 2015;15:25-41.

7. Sawyers CL. The cancer biomarker problem. Nature. 2008;452:54852.

8. Zhao M, He XL, Teng XD.Understanding the molecular pathogenesis and prognostics of bladder cancer: an overview. Chin J Cancer Res 2016;28:92-8.

9. Eguchi S, Yamamoto Y, Sakano S, et al. 2010. The loss of $8 \mathrm{p} 23.3$ is a novel marker for predicting progression and recurrence of bladder tumors without muscle invasion. Cancer Genet Cytogenet 2010;200:16-22.

10. Kruger S, Mess F, Bohle A, et al. Numerical aberrations of chromosome 17 and the 9p21 locus are independent predictors of tumor recurrence in non-invasive transitional cell carcinoma of the urinary bladder. Int J Oncol 2003;23:41-8.

11. Kawauchi S, Sakai H, Ikemoto K, et al. 9p21 index as estimated by dual-color fluorescence in situ hybridization is useful to predict urothelialcarcinoma recurrence in bladder washing cytology. Hum Pathol 2009;40:1783-9.

12. Lamy A, Gobet F, Laurent M, et al. Molecular profiling of bladder tumors based on the detection of FGFR3 and TP53 mutations. J Urol 2006;176:2686-9.

13. Malats N, Bustos A, Nascimento CM, et al. P53 as a prognostic marker for bladder cancer: a meta-analysis and review. Lancet Oncol 2005;6:678-86.

14. Shariat SF, Bolenz C, Godoy G, et al. P53 predictive value for pT1-2 N0 disease at radical cystectomy. J Urol 2009;182:907-13.

15. Shariat SF, Bolenz C, Karakiewicz PI, et al. p53 expression in patients with advanced urothelial cancer of the urinary bladder. BJU Int 2010;105:489-95.

16. Tang K, Wang C, Chen Z, et al. Clinicopathologic and prognostic significance of p21 (Cip1/Waf1) expression in bladder cancer. Int J Clin Exp Pathol 2015;8:4999-5007.

17. Korkolopoulou P, Christodoulou P, Konstantinidou AE, et al. Hum Pathol 2000;31:751-60.

18. Gan X, Lin X, He R, et al. Prognostic and clinicopathological significance of downregulated p16 expression in patients with bladder cancer: a systematic review and meta-analysis. Dis Markers 2016:5259602.

19. Ren B, Li W, Yang Y, et al. The impact of cyclin D1 overexpression on the prognosis of bladder cancer: a meta-analysis. World J Surg Oncol 2014;6;12:55.

20. Lopez-Beltran A, Requena MJ, Luque RJ, et al. Cyclin D3 expression in primary Ta/T1 bladder cancer. J Pathol 2006;209:106-13.
21. Kamai T, Takagi K, Asami H, et al. Decreasing of p27(Kip1)and cyclin $\mathrm{E}$ protein levels is associated with progression from superficial into invasive bladder cancer. Br J Cancer 2001;4;84:1242-51.

22. Mitra AP, Datar RH, Cote RJ. Molecular pathways in invasive bladder cancer: new insights into mechanisms, progression and target identification. J Clin Oncol 2006;24:5552-64.

23. Burger M, Denzinger S, Hartmann A, et al. Mcm2 predicts recurrence hazard in stage $\mathrm{Ta} / \mathrm{T} 1$ bladder cancer more accurately than CK20, Ki67 and histological grade. Br J Cancer 2007;4;96:1711-5.

24. Margulis V, Shariat SF, Ashfaq R, et al. Ki-67 is an independent predictor of bladder cancer outcome in patients treated with radical cystectomy for organ-confined disease. Clin Cancer Res 2006;12:7369-73.

25. Ding W, Gou Y, Sun C, et al. Ki-67 is an independent indicator in non-muscle invasive bladder cancer (NMIBC); combination of EORTC risk scores and Ki-67 expression could improve the risk stratification of NMIBC. Urol Oncol 2014;32:42.e13-9.

26. El-Kott AF, El-baz MA, Mokhtar AA. Proliferating cell nuclear antigen (PCNA) and microvessel density predict survival in the urinary bladder carcinoma. Int Urol Nephrol 2006;38:237-42

27. Karam JA, Lotan Y, Karakiewicz PI, et al. Use of combined apoptosis biomarkers for prediction of bladder cancer recurrence and mortality after radical cystectomy. Lancet Oncol 2007;8:128-36.

28. Bahram GE, Sanati MH, Houshmand M, et al .Expression and prognostic significance of bcl-2 and bax in the progression and clinical outcome oftransitional bladder cell carcinoma. Cell J 2014;15:35663.

29. Yoshimine S, Kikuchi E, Kosaka T, et al. Prognostic significance of $\mathrm{Bcl}-\mathrm{xL}$ expression and efficacy of Bcl-xL targeting therapy in urothelial carcinoma. Br J Cancer 2013;108:2312-20.

30. Karamitopoulou E, Rentsch CA, Markwalder R, et al. Prognostic significance of apoptotic cell death in bladder cancer: a tissue microarray study on 179 urothelial carcinomas from cystectomy specimens. Pathology 2010;42:37-42.

31. Mhawech-Fauceglia P, Cheney RT, Fischer G, et al. FGFR3 and p53 protein expressions in patients with pTa and pT1 urothelial bladder cancer. Eur J Surg Oncol 206;32:231-7.

32. Sung JY, Sun JM, Chang Jeong B, et al. FGFR3 over expression is prognostic of adverse outcome for muscle-invasive bladder carcinoma treated with adjuvant chemotherapy. Urol Oncol 2014;32:49.e23-31.

33. Hernández S, López-Knowles E, Lloreta J, et al. Prospective study of FGFR3 mutations as a prognostic factor in nonmuscle invasive urothelial bladder carcinomas. J Clin Oncol 2006;24:3664-71.

34. Van Rhijn BW, Vis AN, van der Kwast TH, et al. Molecular grading of urothelial cell carcinoma with fibroblast growth factor receptor 3 and MIB-1 is superior to pathologic grade for the prediction of clinical outcome. J Clin Oncol 2003;15;21:1912-21.

35. Naik DS, Sharma S, Ray A, et al. Epidermal growth factor receptor expression in urinary bladder cancer. Indian J Urol 2001;27:208-14.

36. Kim WT, Kim J, Yan C, et al. S100A9 and EGFR gene signatures predict disease progression in muscle invasive bladder cancer patients after chemotherapy. Ann Oncol 2014;25:974-9.

37. Kompier LC, Lurkin I, van der Aa MN, et al. FGFR3, HRAS, KRAS, NRAS and PIK3CA mutations in bladder cancer and their potential as biomarkers for surveillance and therapy. PLoS One 2010;5:e13821.

38. Kim EJ, Kim YJ, Jeong P, et al. Methylation of the RUNX3 promoter as a potential prognostic marker for bladder tumor. J Urol 2008;180;1141-5.

39. Maruyama R, Toyooka S, Toyooka KO, et al. Aberrant promoter methylation profile of bladder cancer and its relationship to clinicopathological features. Cancer Res 2001;61;8659-63.

40. Friedrich MG, Chandrasoma S, Siegmund KD, et al. Prognostic rel- 
evance of methylation markers in patients with non-muscle invasive bladder carcinoma. Eur J Cancer 2005;41:2769-78.

41. Agundez M, Grau L, Palou J, et al. Evaluation of the methylation status of tumour suppressor genes for predicting bacillus Calmette-Guérin response in patients with T1G3 high-risk bladder tumours. Eur Urol 2011;60:131-40.

42. Jäger T, Becker M, Eisenhardt A, et al. The prognostic value of adhering switch in bladder cancer Oncol Rep 2010;23:1125-32.

43. Khorrami MH, Hadi M, Gharaati MR, et al. Expression as a prognostic factor in transitional cell carcinoma of the bladder after transurethral resection. Urol J 2012;9:581-5.

44. Rodriguez Faba 0, Palou-Redorta J, Fernández-Gómez JM, et al. Matrix metalloproteinases and bladder cancer: what is new? ISRN Urol 2012;581539.

45. Miyata Y, Sagara Y, Kanda S, et al. Phosphorylated hepatocyte growth factor receptor/c-Met is associated with tumor growth and prognosis in patients with bladder cancer: correlation with matrix metalloproteinase-2 and -7 and E-cadherin. Hum Pathol 2009;40:496-504.

46. Kopparapu PK, Boorjian SA, Robinson BD, et al. Expression of VEGF and its receptors VEGFR1/NEGFR2 is associated with invasiveness of bladder cancer. Anticancer Res 2013;33:2381-90.

47. El Gehani K, Al-Kikhia L, Mansuri N, et al. Angiogenesis in urinary bladder carcinoma as defined by microvessel density (MVD) after immunohistochemical staining for Factor VIII and CD31. Libyan J Med 2011;31:6.

48. Gust KM, McConkey DJ, Awrey S, et al. Fibroblast growth factor receptor 3 is a rational therapeutic target in bladder cancer. Mol Cancer Ther 2013;121245-54.

49. Milowsky MI, Dittrich C, Durán I, et al. Phase 2 trial of dovitinib in patients with progressive FGFR3-mutated or FGFR3 wild-type advanced urothelialcarcinoma. Eur J Cancer 2014;50:3145-52.

50. Petrylak DP, Tangen CM, Van Veldhuizen PJ Jr, et al. Results of the Southwest Oncology Group phase II evaluation (study S0031) of ZD1839 for advanced transitional cell carcinoma of the urothelium. BJU Int 2010;105:317-21.

51. Philips GK, Halabi S, Sanford BL, et al; Cancer and Leukemia Group B. A phase II trial of cisplatin (C), gemcitabine (G) and gefitinib for advanced urothelial tract carcinoma: results of Cancer and Leukemia Group B (CALGB) 90102. Ann Oncol 2009;20:1074-9.

52. Pruthi RS, Nielsen M, Heathcote S, et al. A phase II trial of neoadjuvant erlotinib in patients with muscle-invasive bladder cancer undergoing radical cystectomy: clinical and pathological results. BJU Int 2010;106:349-54.

53. Strowd RE 3rd, Knovich MA, Lesser GJ. The therapeutic management of bleeding and thrombotic disorders complicating CNS malignancies. Curr Treat Options Oncol 2012;13:451-64.

54. Wong YN, Litwin S, Vaughn D, et al. Phase II trial of cetuximab with or without paclitaxel in patients with advanced urothelial tract carcinoma. J Clin Oncol 2012;30:3545-51.

55. Fransen van de Putte EE, Pos FJ, Bergman A, et al. A phase I study of chemoradiation with weekly panitumumab following neoadjuvant chemotherapy and pelvic lymph node dissection as an organ preserving treatment of invasive bladder cancer. J Clin Oncol 2016;34:abstr 392.
56. Oudard S, Culine S, Vano Y, et al. Multicentre randomised phase II trial of gemcitabine+platinum, with or without trastuzumab, in advanced or metastatic urothelial carcinoma overexpressing HER2. Eur J Cancer 2015;51:45-54.

57. Wülfing C, Machiels JP, Richel DJ, et al. A single-arm, multicenter, open-label phase 2 study of lapatinib as the second-line treatment of patients with locally advanced or metastatic transitional cell carcinoma. Cancer 2009;115:2881-90.

58. Narayan V, Mamtani R, Keefe S, et al. Cisplatin, Gemcitabine, and Lapatinib as Neoadjuvant Therapy for Muscle-Invasive Bladder Cancer. Cancer Res Treat 2016;48:1084-91.

59. Jones R, Hussain SA, Crabb SF, et al. A phase II/II double-blind, randomized trial comparing maintenance lapatinib versus placebo after first line chemotherapy in HER1/2 positive metastatic bladder cancer patients. J Clin Oncol 2015;33:abstr 4505.

60. Chaudhary U, Golshayan A, Brisendine A. Phase II trial of neoadjuvant GC and bevacizumab followed by radical cystectomy (RC) in patients with muscle-invasive transitional cell carcinoma (TCC) of the bladder. J Clin Oncol 2011;29:abstract 276.

61. Balar AV, Apolo AB, Ostrovnaya I, et al. Phase II study of gemcitabine, carboplatin, and bevacizumab in patients with advanced unresectable or metastatic urothelial cancer. J Clin Oncol 2013;31:724-30.

62. Twardowski P, Stadler WM, Frankel P, et al. Phase II study of aflibercept (VEGF-Trap) in patients with recurrent or metastatic urothelial cancer, a California Cancer Consortium Trial. Urology 2010;76:923-6.

63. Petrylak DP, Tagawa ST, Kohli M, et al. Docetaxel as monotherapy or combined with ramucirumab or icrucumab in second-line treatment for locally advanced or metastatic urothelial carcinoma: an open-label, three-arm, randomized controlled phase II trial. J Clin Oncol 2016;34:1500-9.

64. Sonpavde G, Jian W, Liu H, et al. Sunitinib malate is active against human urothelial carcinoma and enhances the activity of cisplatin in a preclinical model. Urol Oncol 2009;27:391-9.

65. Gallagher DJ, Milowsky MI, Gerst SR, et al. Phase II study of sunitinib in patients with metastatic urothelial cancer. J Clin Oncol 2010;28:1373-9.

66. Mullane SA, Bellmunt J. Cancer immunotherapy: new applications in urologic oncology. Curr Opin Urol 2016 [Epub ahead of print].

67. Zibelman M, Plimack ER. Checkpoint inhibitors and urothelial carcinoma: the translational paradigm. Oncology (Williston Park) 2016;30:160-2, 176.

68. Milowsky MI, Iyer G, Regazzi AM, et al. Phase II study of everolimus in metastatic urothelial cancer. BJU Int 2013;112:462-70.

69. Wagle N, Grabiner BC, Van Allen EM, et al. Activating mTOR mutations in a patient with an extraordinary response on a phase I trial of everolimus and pazopanib. Cancer Discov 2014;4:546-53.

70. Gerullis H, Eimer C, Ecke TH, et al. A phase II trial of temsirolimus in second-line metastatic urothelial cancer. Med Oncol 2012;29:2870-6.

71. Apolo AB, Tomita Y, Lee MJ, et al. Effect of cabozantinib on immunosuppressive subsets in metastatic urothelial carcinoma. J Clin Oncol 2014;32:abstr 4501. 